Portland State University

PDXScholar

School of Social Work Faculty Publications and

Presentations

School of Social Work

Fall 2011

\title{
The Social Nature of Male Suicide: A New Analytic Model
}

Daniel Coleman

Portland State University

John T. Casey

Follow this and additional works at: https://pdxscholar.library.pdx.edu/socwork_fac

Part of the Mental and Social Health Commons, Psychiatry and Psychology Commons, and the Social Work Commons

Let us know how access to this document benefits you.

\section{Citation Details}

Coleman, D., Kaplan, M. S., \& Casey, J. T. (2011). The Social Nature of Male Suicide: A New Analytic Model. International Journal Of Men's Health, 10(3), 240-252. doi:10.3149/jmh.1003.240

This Article is brought to you for free and open access. It has been accepted for inclusion in School of Social Work Faculty Publications and Presentations by an authorized administrator of PDXScholar. Please contact us if we can make this document more accessible: pdxscholar@pdx.edu. 


\section{The Social Nature of Male Suicide: A New Analytic Model}

Suicide is one of the leading causes of male mortality. In nearly every country in the world, more males than females end their life by suicide. Previous research indicates male-specific risk factors include social factors such as being unmarried, low income, and unemployment. An analytic model of male suicide is developed, proposing that the traditional male gender role creates a culturally-conditioned narrowing of perceived options and cognitive rigidity when under stress that increases male suicide risk. Suicide prevention and intervention require recognition of the role of high traditional masculinity, situating individual explanations within a broader social context. Based on this theory and the few existing empirical studies, testable hypotheses are proposed.

Keywords: suicide, men, masculinity, gender

This is what my father did, he got up, showered, shaved and dressed for work. He went downstairs and made a pot of coffee, and while it was brewing he went outside and walked the long driveway to pick up the newspaper. He left the paper folded on the kitchen table, poured a cup of coffee, carried it upstairs, and put it on my mother's table. She was still in bed, sleeping. Then he went into his study, closed the door, and shot himself. - Joan Wickersham (2008, p. 5)

Understanding male suicide requires a social lens. This paper first reviews the epidemiology of male suicide and research on risk factors for male suicide. Then an analytic model is developed that interprets male suicide as a function of male gender role extremes, and in light of Baumeister's (1990) escape theory of suicide. As Wickersham's (2008) haunting account of her father's suicide exemplifies (quoted above), many male suicides do not fit a stereotype of being visibly depressed and having made previous attempts. Focus is needed on developing and testing explanatory theories of male suicide, and using this evidence to tailor prevention and intervention programming toward men. The evidence synthesized comes from many cultures, but the diversity in gender roles around the world is great, so this paper serves as a starting point for understanding gender, gender role and patterns of suicide.

Death by suicide is a strikingly a male phenomenon. More men complete suicide every year in every country in the world with one exception, China (Joiner, 2005). In

\footnotetext{
* Portland State University.
}

Correspondence concerning this article should be sent to Daniel Coleman, School of Social Work, P.O. Box 751-SSW, Portland, OR 97207. E-mail: dcoleman@pdx.edu 
the United States, men are more than four times as likely as women to complete suicide, a large effect (CDC, 2009). In Europe, the widest gap between men and women for suicide mortality is found in Greece and Ireland. In England and Wales, men are approximately three times as likely to complete suicide as women (Payne, Swami, \& Stanistreet, 2008). The consistency in epidemiological data showing a male excess in suicide is impressive, but Canetto (2008) cautions that epidemiological data is missing for some developing countries, and there are cultural differences in how deaths are recorded.

Across both genders, suicide is a significant global public health problem. According to World Health Organization estimates, 10-20 million people complete suicide every year. There are wide variations between countries in terms of suicide mortality, with very low rates in some Latin American and Muslim countries, compared with high rates in Eastern Europe. Variations in the way suicide is recorded affect those comparisons, particularly in countries where suicide goes against religious beliefs, but such variations do not fully explain differences between countries (Mann et al., 2005; Payne, 2006; WHO, 2002).

\section{Male Suicide Risk Factors and Precipitating Circumstances}

The gap between women and men in suicide risk varies across the age span in many regions of the world. In Australia, for example, the widest gap between men and women in suicide deaths is found in young adults and among those aged 70 and older. Similarly, in the United States the greatest gender difference is among those aged 15-24 and those over 65. In New Zealand, men are more than three times as likely to complete suicide, but in younger age groups this increases to a four-fold risk (Payne, 2006).

There are additional gender differences in suicide methods (Payne. 2006). More men use lethal methods, including firearms, hanging and jumping. In the United States, firearms are used in nearly 60 percent of male deaths, ranging from 23 percent in Massachusetts to 66 percent in South Carolina. Methods used in suicide also vary with age. Three out of every four suicides among U.S. older adults (65+) involve firearms, compared with just over half of those aged 15-24 (Kaplan, McFarland, \& Huguet, 2009).

Some risk factor studies analyzed male and female participants separately, as well as in aggregate, highlighting the divergent risks for male and female suicide. A large Danish death register study reported that males who are single, unemployed or whose income is lower than average had a heightened risk of suicide, while those same factors were not significant for women (Qin, Agerbo, \& Mortensen, 2003). In a large, nationally representative study in the US, education and marital status other than married were risk factors for suicide death for men but not for women (Denney, Rogers, Krueger, \& Wadsworth, 2009). The time of marital separation has been found to be a particularly vulnerable time for suicide death, with higher risk for males (Wyder, Ward \& De Leo, 2009). Male attempters are more likely to be first-time attempters (Murase, Ochiai, Ueyama, Honjo, \& Ohta, 2003), more likely to be underweight than averageweight (Kaplan, McFarland, Huguet, \& Newsom, 2006; Carpenter, Hasin, Allison, \& Faith, 2000), and more likely to be cigarette smokers and to have stressors related to low income (Zhang, McKeown, Hussey, Thompson, \& Woods, 2004). Kaplan and coauthors (2006) found risk factors for suicide included male gender, physical illness and disability, psychiatric condition, and military veteran status. 
There are both distal and proximal risk factors that are salient to male suicide (Hufford, 2001). Risk factors of a chronic and longstanding nature, such as alcohol dependence or psychiatric disorders, are relatively distal factors, in contrast to proximal factors that have a sudden onset and precipitate a crisis, such as an unexpected loss of job, relationship, or home (Allen, Cross, and Swanner, 2005). While substance dependence is a distal factor, acute alcohol intoxication constitutes a proximal factor that can increase the risk of both a suicide attempt and the use of lethal means (Hufford, 2001). In 17 US states, over one-quarter of male suicide decedents had a blood alcohol concentration indicative of intoxication at the time of death. Additionally, alcohol intoxication was predictive of the use of firearms after adjusting for other variables (Kaplan, McFarland and Hugeut, 2009).

Increased suicide risk is found in several male dominated occupations including police officers, the military, farmers, and physicians. These fields all have access to lethal means and a high likelihood of work related exposure to death. Police, military personnel, and farmers tend to use firearms to complete suicide, and physicians are more likely to use a medication overdose (Agerbo, Gunnell, Bond, Mortensen, \& Nordentoft, 2007).

\section{The Social Environment and Male Suicide}

There has been a longstanding tension between social and individual explanations for suicide, evident as early as Durkheim's (1897/1997) pioneering sociological study of suicide. Durkheim made note of the male excess in suicide, and the majority of examples he explores were of exclusively male or male dominated segments of society such as the military. Dublin (1962), who contributed to the development of demographic methods starting in the early $20^{\text {th }}$ century, also turned his attention to suicide. Canetto (2008) points out when Dublin did attend to gender differences, it was largely informed by stereotypes about men and women. In recent decades, sociology has devoted substantial attention to the issue of suicide (Stack, 2000), but research on the social context and suicide has come from a range of disciplines.

As noted previously, men have higher risk of suicide following job loss than women. According to several reviews, being unemployed is associated with a twofold to threefold increased relative risk of death by suicide, compared with being employed. Both aggregate-level and case-control studies generally show that unemployment is positively correlated with male suicide rates in several Western countries. Based on data drawn from Danish longitudinal registers, Qin et al. (2003) demonstrated that economic stressors such as unemployment and low income increase suicide risk more in male than female subjects. The significant risk factors for men, after controlling for psychiatric admission, were unemployment, retirement, being single and sick absence. For women there were no significant risk factors other than mental illness.

The pattern of male reactivity to unemployment has been found in an impressive series of international studies. Among census-based cohorts of unemployed British and Finnish men, the suicide rates were, respectively, 1.6 and 1.9 times greater than those of the reference populations (Jin, Shah, \& Svoboda, 1995). Time-series analyses also revealed strong aggregate-level correlations between unemployment and suicide among young adult males in Australia (Morrell, Taylor, \& Quine, 1993). Ying and Chang (2009) showed that unemployment had a significantly positive impact on male suicide 
rate but mixed impacts on female suicide rate based on an analysis of panel data from G-7 industrial countries (Canada, France, Germany, Italy, Japan, UK, and US). In a Swedish study involving the impact of job loss on mortality risk, Eliason and Storrie (2009) found that the suicide mortality risk among men increased, while there was no impact on women. Men who lost their jobs were 2.15 times more likely to die by suicide four years after being displaced due to plant closures in Sweden in 1987 and 1988.

There is some evidence that the influence of unemployment varies with age. Berk, Dodd and Henry (2006) reported that the relationship between unemployment and suicide in Australia was strongest for males in the 20-34 year-old age range during the period of 1968-2002. Similarly, when the unemployment rate tripled during the Hong Kong economic crisis of the 1990s, there was a 93\% increase in the rate of suicide death for males in the 30-59 year-old category (Chen, et al, 2006). It is logical that the impact of unemployment would be strongest in work and career sensitive developmental stages.

The influence of economic conditions operates at least partially independently of culture. In the US, the aggregate African-American suicide rate has been consistently markedly lower than the rate for whites. However, the gender discrepancy between African-American men and women is greater than in other groups in the US: AfricanAmerican men are six times more likely to die by suicide than African-American women, and the rate for men has increased in recent decades (Joe \& Kaplan, 2001). Burr, Hartman and Matteson (1999) found that African-American suicide rates were highest in geographical areas that had the greatest income disparities between whites and African-Americans. The overall lower rate of African-American suicide may stem from protective cultural factors such as strong extended family ties, but the excess in male suicide again likely reflects the influence of poverty, unemployment and racism. An additional factor may be gender role orientation within the African-American community.

The impact of historical and current poverty and social dislocation is evident also in the elevated suicide rate for Native Americans, approximately one and a half times the general population US rate. There is a male excess of suicides among Native Americans, as well, peaking with a suicide rate of two and a half times the population rate for Native American men 15-24 years of age (Olson \& Wahab, 2006). These patterns appear to be similar among Aboriginal peoples in Australia (Tait \& Carpenter, 2010) and Canada (Laliberte \& Tousignant, 2009).

In summary, unemployment may act as a proximal stressful life event leading to suicide (Shah \& Bhandarkar, 2008). Economic insecurity may be an important antecedent variable in the causal chain leading a person to attempt suicide (Jin, Shah, \& Svoboda, 1995). The stress associated with unemployment can differ depending on one's options and constraints. In a later section, we argue that the traditional male gender role narrows perceived options under stressful circumstances. Given the connection of male suicidality to social influences, the dominance of individualized therapeutic models of suicidal behavior obscures critical precipitating social circumstances (Moller, 1996).

\section{Suicide and Homophobia}

There is evidence that gay and bisexual adolescent males are at greater risk for suicide attempts and completions (Cochran \& Mays, 2008). Although some investigators 
attribute suicide risk to mental health problems, others have found that the association between suicidality and same-gender orientation in adolescence and adult men is independent of the effects of substance use and mental health diagnoses (Remafedi, 2008). Cochran and Rabinowitz's (2003) analysis of Danish data showed a six-fold increase in age-adjusted risk for completed suicide among men, but not women, who were in registered same-sex domestic partnerships when compared to married persons. Herrell et al. (1999) found that gay men were 6.5 times as likely as their fraternal cotwins to have attempted suicide and the relatively high risk was not explained by mental health or substance abuse disorders. In two large studies of students conducted in Minnesota and Massachusetts, the relative risks of attempted suicide for bisexual and gay male students were, respectively, 7.1 and 3.4 times higher than heterosexual male peers (Remafedi, 2008).

Safren and Heimberg (1999) found similar elevated rates of suicidal behavior for gay and lesbian youth, but when the increased psychosocial stress for these youth was controlled for, the group difference became much smaller. This indicates that much of the difference in suicidal ideation and behavior for sexual minority youth is due to the stress of alienation from friends and family members related to continued homophobia. In addition to the stress of homophobia of others, gay and bisexual men may struggle with internalized homophobia.

\section{Males And Violent Means of Suicide}

As noted earlier, men are more prone to use violent, highly lethal means such as guns, hanging or jumping. Eighty-percent of all suicide deaths in the U.S. involve males, and the majority of those deaths involve the use of firearms (Miller, Lippmann, Azrael, \& Hemenway, 2007). Among elderly men, guns are the most common method of suicide, accounting for nearly $80 \%$ of such deaths (Kaplan et al., 2009).

The use of violent means by male attempters has been interpreted as consistent with the traditional male gender role (Canetto, 1997; Stack \& Wasserman, 2009). Men are more likely to shoot themselves in the head rather than in the body, and are more likely to use rifles or shot guns to complete suicide (Stack \& Wasserman, 2009). This is convergent with Canetto's (1997) summary of research on youth views of suicide and suicide attempts, where youth tended to view attempts as feminine, and suicide completion as masculine.

The male pattern of suicidality also includes impulsive attempts with lethal means, often while under the influence of drugs or alcohol, and in the absence of long term substance abuse or mental health problems. Kaplan and colleagues (2006) statistically classified decedents into those who displayed a long-term risk profile, and those who fit a short term profile. Those in the short term risk group were more likely to have been male, white, and to use firearms to complete suicide. This impulsive type of suicide is the most difficult to prevent, as warning signs are only evident in a brief window before an attempt, and attempts tend to be fatal.

\section{Men And Suicide: Depression And Beyond}

The review of risk factors indicates that men are more likely to develop suicidal behavior following major life transitions, from unemployment, and being single than 
women with the same stressors. The fields of suicide research and prevention have been understandably focused on the link between suicide and depression. Suicidal thoughts and behavior are by definition linked to depression by inclusion in diagnostic criteria (American Psychiatric Association, 2000), and approximately $20 \%$ of people with major depressive disorder report suicidal behavior or thoughts (Borges, et al., 2006). The majority of suicide research is conducted with clinical samples, reinforcing the link of depression and suicide.

The importance of moving beyond a depression-centered view of male suicide came into focus in one of the authors' analysis of the National Violent Death Reporting System (NVDRS) data (Kaplan, McFarland, \& Huguet, 2009). This analysis used suicide death data from 16 states and included information on decedent mental health from proxy data. From the tables in the article, it can be calculated that $62 \%$ of male decedents had no mental health history or diagnosis, in contrast to $42 \%$ of female decedents. Only half as many men (16\%) as women (32\%) had a previous suicide attempt. The typical male suicide decedent had no history of mental health treatment and no previous suicide attempts. Given that men are less likely to seek mental health treatment, a proportion of these men probably had depression or other mental disorders. Our hypothesis is traditional masculinity would account for additional variance in suicidality beyond depression.

From this data it is clear that the majority of male suicide decedents would not have been included in a study of attempters, nor in a study based on a clinical sample. In other words, the majority of male suicide decedents are drawn from a different population than the populations on which much of our knowledge about suicide is based upon, clinical samples and previous attempters. This explains in part how the dynamics of male suicide continue to be overlooked and poorly understood.

\section{Reframing Individual Psychopathology as a Social Problem}

The gender discrepancy of rates of depression among male and female suicide decedents has been noted before, and explained as male decedents are suffering from other psychopathology: such as personality disorders, substance dependence, or an unrecognized male depressive syndrome. We review these arguments as a step toward offering an alternate, socio-cultural lens on male suicide.

The personality disorder argument to explain the non-depressed male suicide is based in studies that retrospectively diagnose decedents. A meta-analytic review of 29 studies of suicide decedents found that male decedents were more likely to fit personality disorder criteria, and female decedents mood disorder criteria (Arsenault-Lapierre, Kim $\&$ Turecki, 2004). Ernst and colleagues (2004) examined cases of suicide decedents who did not meet DSM Axis I criteria, and concluded that there was evidence of underlying psychopathology in nearly all the cases.

The male-depressive syndrome argument is partially convergent with the personality disorder explanation, as the criteria proposed for male depression overlap with personality disorder criteria. For example, Walinder and Rutz (2001) suggest a new diagnostic category of male depression that includes low stress tolerance, acting out behavior, low impulse control and substance abuse. Note that the DSM criteria for borderline and antisocial personalities include anger, impulsivity, and substance abuse. Van Praag (1996) proposed male depression as an "anxiety/aggression-driven" type, 
and identified the overlap with diagnostic criteria and neurotransmitter similarities of the proposed male depression diagnosis and personality disorders.

Recognizing undiagnosed personality problems and correctly identifying depressed men are essential for clinical practice. However, there are neglected social and public health explanations for male depression and suicide. The traits identified by personality disorder diagnosis, and the male depression syndrome, are also consistent with extremes of the male gender role. The emphasis on action, impulsivity, and the acceptability of anger are a part of the social norm of masculinity that sets the context for male suicide. Are these characteristics better accounted for by personality disorders, male depression, or high traditional masculinity? On one level, this is an empirical question that could be tested in a study that includes measures of all these constructs. On another level, it is a philosophical question of specifying a model and choosing variables and level of analysis.

\section{Men and the Escape Theory of Suicide}

Baumeister's (1990) escape theory of suicide, and Hufford's (2001) subsequent elaboration of it in analyzing alcohol use and suicide, provides a helpful framework for male suicide. The theory is at the individual level, but the dynamics described by escape theory reflect consequences of the dominant male gender role. Though Baumeister (1990) did not emphasize gender in his theory, he argued that depression was not a satisfying explanation for the majority of suicidal behavior, and his theory sought to explain both depressed and non-depressed suicidal acts. Stated simply, escape theory proposes that suicidal acts are more likely when an individual is emotionally distressed by a self-perception of failure and this leads to a narrowing cognitive state of limited emotion, attention, and lowered inhibition. The suicidal act is an effort to escape an intolerable view of self and the related negative affect.

This state of cognitive rigidity is particularly salient to understanding male suicide. The male gender role, with its emphasis on a high expectation of strength, providing materially for others, and the acceptability of anger and violence, provides a social script that combines with cognitive rigidity with often fatal consequences. Hufford (2001) termed the empirical evidence for cognitive constriction while intoxicated, "alcohol myopia," and argued alcohol intoxication exacerbated the dynamics identified in escape theory. We suggest that the traditional male gender role contributes its own myopia, increasing male suicide risk. In the next section we review masculinity theory, showing the connection between masculinity and the cognitive rigidity of escape theory.

\section{MAsCUlinity AND SUICIDE}

There is a recent tradition of literature on masculinity growing out of men's studies that dovetails with Baumeister's theory, underscoring men's limited range of options within the areas of self-identity, relationships and capacity for happiness (David \& Brannon, 1976; Mahalik, 1999). Many of these theories take a developmental perspective. Pollack (2006) formulated that boys are first taught to hide their emotions between the ages of 3 and 5 through a "boy code" that rewards toughness, and relies on shame to enforce a prohibition against emotional expression or vulnerability, a condi- 
tion he called "gender straitjacketing." Across the age-span, the straitjacket metaphor is a male-specific example of Baumeister's theory, where cultural gender-role expectations limit males' options when faced with stress, crisis or loss, thus increasing their risk for self-endangering or self-harming behaviors.

The traditional male gender-role, with its values of the pursuit of success, power, emotional control, fearlessness, and self-reliance (Mahalik, 1999), sets the context for male gender role strain (Levant, 1996). The feelings of inadequacy generated by not meeting gender role ideals are exacerbated by the gender role expectations themselves of avoidance of perceived weakness, unacceptability of emotions other than anger, and prohibitions against dependency or seeking help.

A small number of studies have investigated the influence of masculinity on suicide risk. Hunt, Sweeting, Keoghan and Platt (2006) found that for older participants masculinity was associated with lower suicidal thoughts, but more traditional gender role attitudes were associated with higher suicidal thoughts. The authors note a measurement limitation, that their measure of masculinity tapped participants' experience of mastery and leadership and less of the negative extremes of the traditional male gender role such as limited emotional range and difficulty seeking help. A recent analysis of the 1969 cohort of Swedish military conscripts also found higher masculinity was protective against suicide (Mansdotter, Lundin, Falkstedt, \& Hemmingsson, 2009). The measure of masculinity was a crude ranking based on occupation and leisure interests from the original 1969 conscript assessments.

Despite the limitations of these studies, there likely is an association of certain dimensions of masculinity and low suicide risk in healthy and successful men. One widely used measure of gender role orientation, the Extended Personality Attributes Questionnaire (EPAQ), includes a masculine positive scale as well as a masculine negative scale (Helmreich \& Spence, 1981). Perhaps the positive and healthy parts of masculinity can be reclaimed in the social reworking of gender roles. However, it is when there is a failure to meet an expectation- job loss, relationship break-up, perceived professional failure- that the high masculine risk for suicide is exposed. This is consistent with Baumeister's (1990) escape theory process which begins with a perceived personal failure. Similarly, Mansdotter and co-authors (2009) argued that in their study, the higher rate of suicide for the low masculinity group could be contributed to by the "...stress of not meeting high masculinity ideals..." (p. 412).

A qualitative study of depressed men converges with the two quantitative studies, and reinforces some of the central arguments of this paper. Oliffe and colleagues (2010) interviewed 30 depressed men, with focused attention to suicide and masculinity. They found that men were drawn away from suicide by involvement in some masculine roles such as positive dimensions of fathering. Other men fled into masculine-associated pseudo-independence by pulling away from significant others and increasing alcohol and drug use, reporting increased suicidal thoughts. This is similar to the connection proposed between escape suicide dynamics and traditional masculinity.

\section{DisCUSSION}

Suicide is largely a male phenomenon and has a distinct profile: male decedents are less likely to be depressed or have received mental health services, less likely to have made previous attempts, and more likely to use highly lethal means. Male suicide often 
follows job loss, business failure, relationship loss, or an embarrassing public disclosure. Most of the efforts to explain male suicide focus on individual and psychological explanations, such as unrecognized psychopathology or a male depressive syndrome. In their emphasis on constricted emotion, anger and impulsivity, these explanations reflect the prevailing male gender role. It is important to recognize that a group of male decedents who could be diagnosed with "male depression" are men who have grown up immersed in male socialization. As Moller (1996) stated: “...the psychosocial collage of suicide is comprised of structural conditions, of the psychological state of the individual, and of the manner in which structural or social forces converge with the personal state of the individual and define particular life situations for individuals" ( $\mathrm{p}$. 199). The understanding of male suicide has focused inordinate attention on individual deficits, while neglecting the power of precipitating social circumstances.

Baumeister's (1990) escape theory applies well to male suicide. Escape theory depicts the individual struggling with some injury to self-esteem, and shifting into a crisis mode where the cognitive awareness of options narrows. Hufford (2001) drew the analogy that alcohol use exacerbated this narrowing of options and cognitive rigidity, what he termed "alcohol myopia." Higher degrees of traditional masculinity are likely a risk factor for an "escape suicide" much like acute alcohol use. We propose there may be a "masculinity myopia" that increases risk of suicide. As noted earlier, there are many cultures where we hypothesize this theory is applicable, however this hypothesis should be explored theoretically and empirically in a wide diversity of cultures.

In addition to a theoretical exploration, this paper is a call to action to take on the contribution of the traditional male gender role in suicidal behavior. The negative interpersonal and social effects of the exaggerated social norm of masculinity found in many cultures are clear: namely, an absence of empathy and emotions other than anger, misogyny, homophobia, violence and an emphasis on competition and dominance in interpersonal relationships. Part of the social influence of feminism is freeing the experience of being a woman from constricting social conceptions of femininity. Men have benefited from the reciprocal influence of changing female gender roles exerting an influence on traditional male roles, but in most of the world's societies there has not been a strong or direct focus on male gender roles.

If men are dying of an extreme of the male gender role, the implication is that male suicide prevention should be pursued through tackling those dimensions of the male gender role that increase suicide risk. At the broadest level, this suggests changing the meaning and experience of being a man from the current norm in most societies. An example of this broad view is found in Payne and co-authors' (2008) proposal that reducing suicidal behaviors will require a public health strategy that involves "...a deconstruction of the power structures that give rise to inequalities between men and women" (p. 33). Of course, power structures and social roles change slowly and haltingly, so this goal requires a long term perspective. More narrowly and immediately, public awareness and suicide prevention efforts could be tested that are tailored to the social dynamics of male suicide risk.

An example of a tailored public awareness campaign focused on mental health, not just suicide, is a recent United States Veteran's Administration campaign that centers on the phrase: "It takes the courage and strength of a warrior to ask for help" (www.realwarriors.net). This campaign attempts to work within the masculine concept of the warrior, and to paradoxically use it to increase rather than impede help-seeking be- 
havior. We are ambivalent about working within the stereotyped gender role, and therefore reinforcing it, though we understand the immediacy of trying to break down barriers to help-seeking for veterans and active duty military.

Another example is suicide gatekeeper training, the most popular suicide prevention strategy. The most widely-used of the trainings is "Question, Persuade, Refer" (QPR; www.qprinstitute.com). The typical format of QPR is a two-hour didactic training, targeting knowledge about suicide and suicide risk factors, breaking down myths about suicide, and coaching participants to ask people at risk about suicide and get them to professional help. QPR training involves little or no gender specific information, and centers on identifying depression and the link of depression and suicide, missing the male suicide pattern we have identified in this paper. A version of QPR could be developed that includes more information on gender differences and suicide, or could focus on male suicide for gatekeepers working in primarily male settings such as corrections or the military.

In many healthcare settings there is limited training in identifying and intervening in suicide generally, let alone male suicide. The recognition of suicidal risk among males is often limited by healthcare services that are more adapted to treating females, particularly in mental health and psychiatric settings (Rutz \& Rihmer, 2007).

This paper proposes a hypothesis that male suicide is in part a function of extremes of the traditional male gender role. While we built this argument synthesizing data from a wide range of sources, the hypothesis should be directly tested. The few existing studies found that positive dimensions of masculinity such as leadership and mastery were protective against suicidal ideation and attempts. As Hunt (2006) suggested, a study is needed that includes a measure of the extreme of masculinity that includes constricted affect, inhibitions against help-seeking, and acceptability of anger and violence. Further, our theoretical perspective suggests high traditional masculinity is particularly a risk factor for men who are thwarted in efforts to maintain a masculine ideal by events such as job loss, illness or disability.

In conclusion, the epidemiology of male suicide is stark: men account for four out of five suicides in the United States, use highly lethal means, often complete suicide in their first attempt, and do not signal their risk to others through traditional symptoms of depression. The unique dynamics of male suicide merit attention in research, prevention and treatment efforts. In particular, high traditional masculinity in men experiencing psychosocial stress in work or family life, combined with easy access to lethal means, contributes to an epidemic of male suicide. We look forward to the field producing research to better understand masculinity and male suicide, developing and testing public health and clinical interventions based on this evolving understanding, and hopefully a changing social definition of men and their role in society.

\section{REFERENCES}

Agerbo, E., Gunnell, D., Bond, J.P., Mortensen, P.B., \& Nordentoft, M. (2007). Suicide and occupation: The impact of socioeconomic, demographic and psychiatric differences. Psychological Medicine, 37, 1131-1140.

Allen, J.P., Cross, G., \& Swanner, J. (2005). Suicide in the army: A review of current information. Military Medicine, 170, 580-584. 
American Psychiatric Association (APA). (2000). Diagnostic and statistical manual of mental disorders: DSM-IV-TR. Washington, DC: APA.

Arsenault-Lapierre, G., Kim, C., \& Turecki, G. (2004). Psychiatric diagnoses in 3275 suicides: A meta-analysis. BMC Psychiatry, 4, 1-11.

Baumeister, R.F. (1990). Suicide as escape from self. Psychological Review, 97, 90-113.

Berk, M., Dodd, S., \& Henry, M. (2006). The effect of macroeconomic variables on suicide. Psychological Medicine, 36, 181-189.

Borges, G., Angst, J., Nock, M.K., Ruscio, A.M., Walters, E.E., \& Kessler, R.C. (2006). A risk index for 12-month suicide attempts in the National Comorbidity Survey Replication (NCSR). Psychological Medicine, 36, 1747-1757.

Burr, J.A., Hartman, J.T., \& Matteson, D.W. (1999). Black suicide in U.S. metropolitan areas: An examination of the racial inequality and social integration-regulation hypotheses. Social Forces 77, 1049-1081.

Canetto, S.S. (1997). Meanings of gender and suicidal behavior during adolescence. Suicide and Life-Threatening Beahvior, 27, 339-351.

Canetto, S.S. (2008). Women and suicidal behavior: a cultural analysis. American Journal of Orthopsychiatry, 78(2), 259-266.

Carpenter, K.M., Hasin, D.S., Allison, D.B., \& Faith, M.S. (2000). Relationships between obesity and DSM-IV major depressive disorder, suicide ideation, and suicide attempts: Results from a general population study. American Journal of Public Health, 90, 251-257.

Center for Disease Control and Prevention. (2009). Fatal injury reports. Retrieved October 10, 2009, from http://www.cdc.gov/ncipc/wisqars.

Chen, H., Eric, Y., Chan, C., Wincy, S., Wong, C., Paul, W., et al. (2006). Suicide in Hong Kong: A case-control psychological autopsy study. Psychological Medicine, 36, 815-825.

Cochran, S.D., \& Mays, V.M. (2008). Prevalence of primary mental health morbidity and suicide symptoms among gay and bisexual men. In R.J. Wolitski, R. Stall, \& R.O. Valdiserri (Eds.), Unequal opportunity: Health disparities affecting gay and bisexual men in the United States (pp. 97-120). New York: Oxford University Press.

Cochran, S.V., \& Rabinowitz, F.E. (2003). Gender-sensitive recommendations for assessment and treatment of depression in men. Professional Psychology: Research and Practice, 34,132140.

David, D., \& Brannon, R. (Eds.). (1976). The forty-nine percent majority: The male sex role. Reading, MA: Addison-Wesley.

Denney, J., Rogers, R., Krueger, P., \& Wadsworth, T. (2009). Adult suicide mortality in the United States: Marital status, family size, socioeconomic status, and differences by sex. Social science quarterly, 90(5), 1167-1185.

Dublin, L. (1963). Suicide; A sociological and statistical study. New York: Ronald Press.

Durkheim, E. (1897/1997). Suicide: A study in sociology (J. Spaulding, Trans.; G. Simpson, Ed.). New York: Free Press.

Eliason, M., \& Storrie, D. (2009). Does job loss shorten life? Journal of Human Resources, 44 : 227-302.

Ernst, C., Lalovic, A., Lesage, A., Seguin, M., Tousignant, M., \& Turecki, G. (2004). Suicide and no axis I psychopathology. BMC Psychiatry, 4, 1-5.

Herrell, R., Goldberg, J., \& True, W.R. (1999). Sexual orientation and suicidality: A co-twin control study in adult men. Archives of General Psychiatry, 56, 867-874.

Hufford, M. R. (2001). Alcohol and suicidal behavior. Clinical Psychology Review, 21, 797-811. 
Hunt, K., Sweeting, H., Keoghan, M., \& Platt, S. (2006). Sex, gender role orientation, gender role attitudes and suicidal thoughts in three generations: A general population study. Social Psychiatry and Psychiatric Epidemiology, 41, 641-647.

Jin, R.L., Shah, C.P., \& Svoboda, T.J. (1995). The impact of unemployment on health: A review of the evidence. Canadian Medical Association Journal, 153, 529-540.

Joe, S., \& Kaplan, M. (2001). Suicide among African-American men. Suicide and Life-Threatening Behavior, 31(Suppl.), 106-121.

Joiner, T. (2005). Why people die by suicide. Cambridge, MA: Harvard University Press.

Kaplan, M.S., McFarland, B.H., \& Huguet, N. (2009). Firearm suicide among veterans in the general population: Findings from the National Violent Death Reporting System. The Journal of Trauma, 67, 503-507.

Kaplan, M.S., McFarland, B.H., Huguet, N., \& Newsom, J.T. (2006). Sooner versus later: Factors associated with temporal sequencing of suicide. Suicide and Life-Threatening Behavior, 36, 377-385.

Laliberté, A., \& Tousignant, M. (2009). Alcohol and other contextual factors of suicide in four aboriginal communities of Quebec, Canada. Crisis: The Journal of Crisis Intervention and Suicide Prevention, 30(4), 215-221.

Lee, C., \& Owens, R.G. (2002). The psychology of men's health. Buckingham UK: Open University Press.

Levant, R.F. (1996). The new psychology of men. Professional Psychology: Research and Practice, 27, 259-265.

Mahalik, J.R. (1999). Incorporating a gender role strain perspective in assessing and treating men's cognitive distortions. Professional Psychology: Research and Practice, 30, 333-340.

Mann, J.J., Apter, A., Bertolote, J., Beautrais, A., Currier, D., Haas, A., et al. (2005). Suicide prevention strategies: A systematic review. Journal of the American Medical Association, 294, 2064-2074.

Mansdotter, A., Lundin, A., Falkstedt, D., \& Hemmingsson, T. (2009). The association between masculinity rank and mortality patterns: A prospective study based on the Swedish 1969 conscript cohort. Journal of Epidemiology and Community Health, 63, 408-413.

Miller, M., Lippmann, S.J., Azrael, D., \& Hemenway, D. (2007). Household firearm ownership and rates of suicide across the 50 United States. Journal of Trauma, Injury, and Critical Care, 62, 1029-1035.

Moller, D.W. (1996). Confronting death: Values, institutions and human mortality. New York: Oxford University Press.

Moller-Leimkuhler, A.M. (2003). The gender gap in suicide and premature death or: Why are men so vulnerable? European Archives of Psychiatry and Clinical Neuroscience, 253, 1-8.

Morrell, S., Taylor, R., Quine, S. et al. (1993). Suicide and unemployment in Australia. Social Science and Medicine, 36, 749-756.

Murase, S., Ochiai, S., Ueyama, M., Honjo, S., \& Ohta, T. (2003). Psychiatric features of seriously life-threatening suicide attempters: A clinical study from a general hospital in Japan. Journal of Psychosomatic Research, 55, 379-383.

Oliffe, J., Ogrodniczuk, J., Bottorff, J., Johnson, J., \& Hoyak, K. (2010). You feel like you can’t live anymore. Social Science \& Medicine. DOI: 10.1016/j.socscimed.2010.03.057

Olson, L., \& Wahab, S. (2006). American Indians and suicide. Trauma, Violence, \& Abuse, 7(1), 19-33.

Payne, S., Swami, V., \& Stanistreet, D.L. (2008). The social construction of gender and its influence on suicide: A review of the literature. Journal of Mental Health, 5, 23-35. 
Payne, S. (2006). The health of men and women. Cambridge UK: Polity Press.

Pollack, W.S. (2006). The 'war' for boys: Hearing 'real boys" voices, healing their pain. Professional Research and Practice, 37, 190-195.

Qin, P., Agerbo, E., \& Mortensen, P.B. (2003). Suicide risk in relation to socioeconomic, demographic, psychiatric, and familial factors: A national register-based study of all suicides in Denmark, 1981-1997. American Journal of Psychiatry, 160, 765-772.

Remafedi, G. (2008). Health disparities for homosexual youth: The children left behind. In R.J. Wolitski, R. Stall, \& R.O. Valdiserri (Eds.), Unequal opportunity: Health disparities affecting gay and bisexual men in the United States (pp. 275-302) . New York: Oxford University Press.

Rutz, W., \& Rihmer, Z. (2007). Suicidality in men - Practical issues, challenges, solutions. Journal of Men's Health and Gender, 4, 393-401.

Safren, S.A., \& Heimberg, R.G. (1999). Depression, hopelessness, suicidality, and related factors in sexual minority and heterosexual adolescents. Journal of Consulting and Clinical Psychology, 67, 859-866.

Shah, A., \& Bhandarkar, R. (2008). Cross-national study of the correlation of general population suicide rates with unemployment rates. Psychological Reports, 103, 793-796.

Stack, S. (2000). Suicide: A 15-year review of the sociological literature. Part I: cultural and economic factors. Suicide \& life-threatening behavior, 30(2), 145-162.

Stack, S., \& Wasserman, I. (2009). Gender and suicide risk: The role of wound site. Suicide and Life-Threatening Behavior, 39, 13-20.

Tait, G., \& Carpenter, B. (2010). Firearm suicide in Queensland. Journal of Sociology, 46(1), 8398.

van Praag, H.M. (1996). Faulty cortisol/serotonin interplay: Psychopathological and biological characterisation of a new, hypothetical depression subtype (SeCA depression). Psychiatry Research, 65, 143-157.

Walinder, J., \& Rutz, W. (2001). Male depression and suicide. International Clinical Psychopharmacology, 16(Suppl. 2), S21-S24.

Wickersham, J. (2008). The suicide index: Putting my father's death in order. Orlando, FL: Harcourt Books.

World Health Organization (WHO). (2006). Suicide. Retrieved October 10, 2009, from http://www.who.int/topics/suicide/en.

Wyder, M., Ward, P., \& De Leo, D. (2009). Separation as a suicide risk factor. Journal of Affective Disorders, 116(3), 208-213.

Ying, Y.H., \& Chang, K. (2009). A study of suicide and socioeconomic factors. Suicide and LifeThreatening Behaviors, 39, 214-226.

Zhang, J., McKeown, R.E., Hussey, J.R., Thompson, S.J., \& Woods, J.R. (2004). Gender differences in risk factors for attempted suicide among young adults: Findings from the third national health and nutrition examination survey. Annals of Epidemiology, 15, 167-174. 
Copyright of International Journal of Men's Health is the property of Men's Studies Press and its content may not be copied or emailed to multiple sites or posted to a listserv without the copyright holder's express written permission. However, users may print, download, or email articles for individual use. 